\title{
Optical linear polarization in ultra cool dwarfs
}

\section{A tool to probe dust in the ultra cool dwarf atmospheres}

\author{
R. Tata ${ }^{1}$, E. L. Martín ${ }^{2,3,1}$, S. Sengupta ${ }^{4,5}$, N. Phan-Bao ${ }^{6,7}$, M. R. Zapatero Osorio ${ }^{2,3}$, and H. Bouy ${ }^{3}$ \\ 1 University of Central Florida, Department of Physics, Orlando, 32816-2385, USA \\ e-mail: tata@physics.ucf.edu \\ 2 Centro de Astrobioloíga (CAB-CSIC), Ctra. Ajalvir km 4, 28850 Torrejón de Ardoz, Madrid, Spain \\ Instituto de Astrofísica de Canarias, 38205 La Laguna, Tenerife, Spain \\ Indian Institute of Astrophysics, Koramangala, Bangalore, 560034 India \\ TIARA-ASIAA/National Tsing Hua University, Hsinchu, Taiwan \\ ${ }^{6}$ Institute of Astronomy and Astrophysics, Academia Sinica, PO Box 23-141, Taipei 106, Taiwan, China \\ 7 Department of Physics, HCMIU, Vietnam National University Administrative Building, Block 6, Linh Trung Ward, \\ Thu Duc District, HCM, Vietnam
}

Received 11 February 2009 / Accepted 13 October 2009

\section{ABSTRACT}

\begin{abstract}
Aims. Recent studies have detected linear polarization in L dwarfs in the optical $I$ band. Theoretical models have been developed to explain this polarization. These models predict higher polarization at shorter wavelengths. We discuss the polarization in the $R$ and $I$ band of 4 ultra cool dwarfs.

Methods. We report linear polarization measurements of 4 ultra cool dwarfs in the $R$ and $I$ bands using the Intermediate dispersion Spectrograph and Imaging System (ISIS) mounted on the $4.2 \mathrm{~m}$ William Herschel Telescope (WHT).

Results. As predicted by theoretical models, we find a higher degree of polarization in the $R$ band when compared to polarization in the $I$ band for $3 / 4$ of these ultra cool dwarfs. This suggests that dust scattering asymmetry is caused by oblateness . We also show how these measurements fit the theoretical models. A case for variability of linear polarization is found, which suggests the presence of randomly distributed dust clouds. We also discuss one case for the presence of a cold debris disk.
\end{abstract}

Key words. polarization - atmospheric effects - stars: low-mass, brown dwarfs

\section{Introduction}

A large number of ultra cool dwarfs have been detected in the last decade, and our understanding of these faint objects has kept improving. One of the challenging and fundamental aspects in the study of these objects is to understand the properties and distribution of condensate dust in the atmosphere. Observations of L dwarfs with effective temperatures of 1400-2200 K have led to the investigation of dust condensates in their atmospheres (Kirkpatrick et al. 1999; Tsuji et al. 1996). Because of complete gravitational settling, grains are expected to condense beyond the visible atmosphere for objects with effective temperatures below $1400 \mathrm{~K}$ (T-Dwarfs - Allard et al. 2001; Chabrier et al. 2000). At higher effective temperatures (1400-2200 K), grains can be present in the visible atmosphere because of incomplete gravitational settling (Burrows \& Sharp 1999; Burrows et al. 2001; Ackerman \& Marley 2001; Allard et al. 2001; Tsuji et al. 2004; Cooper et al. 2003; Helling et al. 2008). Recent discoveries of blue L dwarfs and L-T transition type dwarfs (as identified in Knapp et al. 2004; Chiu et al. 2006; Tsuji \& Nakajima 2003) have brought forth models which could explain this phenomenon (e.g. Burrows et al. 2006; Knapp et al. 2004) by mechanisms which involve dust settling. It would be very important to validate these mechanisms.

Linear polarization could be a very useful tool in understanding the observationally poorly constrained dust properties in the atmospheres of $\mathrm{L}$ dwarfs. The possibility of detecting polarization at optical wavelengths from grains in the atmospheres of L dwarfs was first raised by Sengupta \& Krishan (2001). Fast rotation of $\mathrm{L}$ dwarfs will induce the shape of their photosphere into the form of an oblate ellipsoid (Basri et al. 2000) and this nonsphericity will lead to the incomplete cancellation of the polarization from different areas of the stellar surface (Sengupta \& Krishan 2001). This prediction was first confirmed by the detection of linear polarization at $768 \mathrm{~nm}$ from a few L dwarfs by Ménard et al. (2002). Recently, Zapatero Osorio et al. (2005) have reported $R$ and $I$ band detection of linear polarization from several L dwarfs. Since polarization in the optical is unlikely to be due to Zeeman splitting of atomic or molecular lines or by synchrotron radiation, the observed polarization can be explained by single dust scattering in a rotationally induced oblate atmosphere (Sengupta 2003; Sengupta \& Kwok 2005) or it could be due to large and randomly distributed dust clouds (Ménard et al. 2002).

In this paper, we report polarization measurements of 3 L dwarfs (L0-L5) and one M9.5 dwarf with WHT/ISIS in both $I$ and $R$ bands. We also discuss our results comparing them with the recently published results of Goldman et al. (2009).

Our measurements show the general trend that polarization is higher in the $R$ band than the one in the $I$ band. This trend strongly supports the presence of dust in the atmosphere of $\mathrm{L}$ dwarfs as it is very unlikely that any other mechanisms (such 
Table 1. Target list.

\begin{tabular}{|c|c|c|c|c|c|c|c|c|c|}
\hline Object & RA & Dec. & $S p T$ & $\begin{array}{l}R \\
(\mathrm{mag})\end{array}$ & $\begin{array}{l}I \\
(\mathrm{mag})\end{array}$ & $\begin{array}{l}J \\
\text { (mag) }\end{array}$ & $\begin{array}{l}d \\
(\mathrm{pc})\end{array}$ & $\begin{array}{l}\mathrm{H} \alpha \\
E W(\AA)\end{array}$ & Ref. \\
\hline 2MASSW J1438082+640836 & 143808.2 & +640836 & M9.5 & & 16.8 & 12.92 & $7.2 \pm 0.5$ & -2.36 & 1 \\
\hline 2MAS & 150747.69 & -162738.6 & L5 & 18.9 & 16.5 & 12.8 & $7.33 \pm 0.03$ & -5.01 & $3,4,5$ \\
\hline 2MASS J17312974+2721233 & 173129.74 & +272123.3 & L0 & $\cdots$ & & 12.09 & $11.80 \pm 0.70$ & -5.98 & 1,2 \\
\hline 2MASSI J1807159+501531 & 180715.93 & +501531.6 & L1.5 & $\ldots$ & & 12.96 & $14.6 \pm 1.0$ & -1.38 & $1,2,3$ \\
\hline
\end{tabular}

Note: Ref.- 1. Schmidt et al. (2007), 2. Jameson et al. (2007), 3. Cruz et al. (2003), 4. Reid, et al. (2000), 5. Knapp et al. (2004). H $\alpha$ EW from Schmidt et al. (2007).

as the presence of magnetic field) can explain this observation at optical wavelengths (Ménard et al. 2002). We also discuss how the theoretical models (see Sect. 3) successfully fit our measured data.

\section{Target selection and observations}

Four very nearby $(7 \mathrm{pc}-15 \mathrm{pc})$ ultra cool dwarfs were selected (SpT M9.5 - L5) as they are among the brightest and nearest ultra cool dwarfs with no known infrared excess and no evidence of multiplicity (see Table 1). These selection criteria ensure that the targets are bright enough sources in the $R$ band to get high $\mathrm{S} / \mathrm{N}$ and to avoid other than intrinsic sources of polarization such as circumstellar disks or multiplicity. For calibration, one polarized (Cyg OB2 A Whittet et al. 1992) and one unpolarized standard star were observed at two different times during the night. All the objects were observed in a way that they insured they were acquired at the same position on the detector ( 5 pixel box). This procedure minimized contamination caused by instrumental polarization within the detector and variations in the optical path.

The polarimetric observations were obtained using the Intermediate dispersion Spectrograph and Imaging System (ISIS) which is mounted at the Cassegrain focus of the $4.2 \mathrm{~m}$ William Herschel Telescope (located in La Palma, Canary Islands, Spain). ISIS in polarization mode is a modulation polarimeter with a double-beam analyzer (the calcite plate) and a rotating halfwave plate modulator. ISIS is equipped with two detectors: a blue-sensitive EEV12 $(4096 \times 2048$ pixels $)$ and a redsensitive RED+ $(4096 \times 2048)$ detector. In our program, we have used the RED+ detector.

Images were obtained using Bessel $R$ - and $I$-filters centered on 641 and $812 \mathrm{~nm}$, respectively, on June 18, 2006 (UT Date). The night was photometric with stable average seeing of 1.0 arcsec.

The raw images were bias-subtracted and flat-fielded before performing aperture photometry. The flat-field images were obtained with the polarimeter optics. Fluxes were obtained for 0.8 , $1.0,1.2,1.5,2.0$ times the average $F W H M$ for each object. The best aperture was chosen to be 1.5 times $F W H M$ based on minimum photon contribution of nearby sources, variable sky contribution, and maximum signal-to-noise ratio of the measurements. The average $F W H M$ of all images was 4.0 pixels which corresponds to 1.0 arcsec. We have only one set of measurements for each object. Therefore, we have estimated the uncertainty in the degree of polarization from various apertures (a similar method was used by Zapatero Osorio et al. (2005) for some of the objects). There was no significant instrumental polarization found as the unpolarized standard measured $D(p)=0.086 \% \pm 0.002$.

\section{Theoretical modeling of polarization}

Polarization is a measure of anisotropy in the radiation field and is caused by either scattering or is due to the presence of magnetic field. The state of the polarization of light is described by the Stokes parameters, $I, Q, U$ and $V$. The parameter $I$ is the total scalar specific intensity of radiation. It is the complete flux of radiant energy inside the unit intervals of frequency, time, solid angle, and area perpendicular to the flux. This flux includes all radiation independently of polarization. Polarization is described by the parameters $Q, U, V$. These parameters are proportional to the scalar specific intensity and have the same dimension. $Q$ and $U$ represent the linearly polarized component, and $V$ represents the circularly polarized component. For linear polarization, $V=0$ and the degree of polarization is given as $p=\sqrt{Q^{2}+U^{2}} / I$. If we consider axial symmetry, then $U=0$ and in that case we define the degree of polarization $p=-Q / I$. The sign convention is such that if $p>0$, the light is polarized perpendicular to the scattering plane, and if $p<0$, the light is polarized parallel to the scattering plane. For an unresolved stellar object, the Stokes parameters are integrated over the stellar disk.

From our obtained images, the degree of polarization and the polarization angles are calculated using the following equations:

$$
\begin{aligned}
& R_{Q}^{2}=\frac{o\left(0^{\circ}\right) / e\left(0^{\circ}\right)}{o\left(45^{\circ}\right) / e\left(45^{\circ}\right)} ; Q / I=\frac{R_{Q}-1}{R_{Q}+1} \\
& R_{U}^{2}=\frac{o\left(22.5^{\circ}\right) / e\left(22.5^{\circ}\right)}{o\left(67.5^{\circ}\right) / e\left(67.5^{\circ}\right)} ; U / I=\frac{R_{U}-1}{R_{U}+1} \\
& p=\sqrt{(Q / I)^{2}+(U / I)^{2}} \\
& \theta=0.5 \tan ^{-1} \frac{U / I}{Q / I}
\end{aligned}
$$

where

$o, e \quad$ ordinary and extraordinary fluxes respectively of the dual images of the program source on a single frame;

$p \quad$ degree of polarization;

$\theta \quad$ polarization angle;

$I \quad$ the total scalar specific intensity of radiation;

$Q / I, U / I$ normalized Stokes parameters.

As pointed out by Ménard et al. (2002), the observed linear polarization in the optical cannot be due to magnetic field, and scattering remains the most viable mechanism for yielding the detected linear polarization. Polarimetric observation at the $R$ and $I$ bands by Zapatero Osorio et al. (2005) shows that polarization decreases significantly with the increase in wavelength, which strongly supports this argument (Sengupta \& Kwok 2005). In the present investigation, we report detection of polarization at both 
$R$ and $I$ bands which shows the same wavelength dependency and hence strengthens the case for scattering polarization. If the dust density is low, then the single scattering approximation is reasonable for the region where the dust optical depth $\tau_{d}<1$ because scattering by atoms and molecules does not significantly contribute to polarization. However, in the optical region $\tau_{d}$ is much higher than 1, and therefore multiple scattering is the appropriate process. Multiple scattering reduces the amount of polarization significantly because the planes of scattering are oriented randomly and cancel each others' contributions. In the present paper, however, we adopt a simple single scattering polarization model because the main aim is to explain the wavelength dependency of the observed polarization. Nevertheless, we find that a single dust scattering model in rotation-induced oblate L dwarfs can fit the observed data well within the permissible range of parameter space of dust composition, size, geometrical distribution and rotation period. A detailed modeling with multiple scattering is in progress.

The simple theoretical model adopted here to explain the observed polarization is described in details in Sengupta \& Kwok (2005). At an edge-on view, the degree of polarization integrated over the stellar disk is given by:

$$
\begin{aligned}
p(\lambda)= & \frac{\lambda^{2}}{g} \int_{P_{\text {top }}}^{P_{\text {bot }}} \frac{n(P) \mathrm{d} P}{\rho(P)} \\
& \times \sum_{l=2}^{\infty}\left\{\alpha^{2}(l, m) P_{l}^{m}(0) F_{l 2} \int_{-1}^{1} \frac{P_{l}(\mu)}{\left[1+\left(A^{2}-1\right) \mu^{2}\right]^{1 / 2}} \mathrm{~d} \mu\right\}
\end{aligned}
$$

In the above expression, $P$ is the total pressure (gas plus dust), $P_{\text {top }}$ and $P_{\text {bot }}$ are the pressures at the top (deck) and the bottom (base) of the dust layer, $n(P)$ is the grain number density at different pressure heights, $\rho(P)$ is the total density at any pressure level, and $A$ is the ratio of the equatorial radius to the polar radius. Since the dust density is small compared to the gas density, $\rho(P)$ is the effective gas density and is equal to $n R T / P$ where $n$ is the mean molecular weight and $R$ is the gas constant. In the above equation, $P_{l}(\mu)$ is the Lengendre polynomial where $\mu=\cos \theta, \theta$ being the scattering angle and $F_{l} 2$ is given by

$F_{l m}=\alpha(l, m) \int_{-1}^{1} \frac{i_{1}-i_{2}}{2} P_{l}^{m}(\cos \theta) \mathrm{d}(\cos \theta)$.

where

$\alpha(l, m)=\left[\frac{(2 l+1)(l-m)}{4 \pi(l+m)}\right]^{1 / 2}$,

and $P_{l}^{m}$ is the associated Legendre function of the first kind. $i_{1}$ and $i_{2}$ are the scattering functions given by van de Hulst (1957).

The vertical dust distribution and the location of the cloud base and deck in the atmosphere are calculated based on the one dimensional heterogeneous cloud model of Cooper et al. (2003). This model assumes chemical equilibrium throughout the atmosphere and uniform density distribution across the surface of an object at each given pressure and temperature. The number density of cloud particles in this model is given by

$n(P)=q_{\mathrm{c}}\left(\frac{\rho}{\rho_{\mathrm{d}}}\right)\left(\frac{\mu_{\mathrm{d}}}{\mu}\right)\left(\frac{3}{4 \pi a^{3}}\right)$,

where $\rho$ is the mass density of the surrounding gas, $a$ is the cloud particle radius, $\rho_{\mathrm{d}}$ is the mass density of the dust condensates, $\mu$ and $\mu_{\mathrm{d}}$ are the mean molecular weight of atmospheric gas and condensates respectively. The condensate mixing number ratio $\left(q_{c}\right)$ is given as

$q_{\mathrm{c}}=q_{\text {below }} \frac{P_{\mathrm{c}, l}}{P}$

for heterogeneously condensing clouds. In the above equation, $q_{\text {below }}$ is the fraction of condensible vapor just below the cloud base, $P_{\mathrm{c}, l}$ is the pressure at the condensation point.

A log-normal size distribution is adopted for the spherical grains (Ackerman \& Marley 2001). The formation of dust makes it a prohibitive task to develop a fully consistent atmospheric model for ultra-cool dwarfs. This is mainly because of the fact that the presence of dust clouds affects the radiative equilibrium of the upper atmosphere and hence alters the T-P profile from that of a cloud-free atmosphere. On the other hand, the T$\mathrm{P}$ profile dictates the position and the chemical equilibrium of condensates. Allard et al. (2001) presented atmospheric models for two of the limiting cases, e.g., one with inefficient gravitational settling wherein the dust is distributed according to chemical equilibrium predictions (AMES-dusty) and another with efficient gravitational settling in which situation dust has no effect on the thermal structure (AMES-cond). Tsuji et al. (2004) have proposed a Unified Cloudy Model (UCM) in which the segregation of dust from the gaseous mixture takes place in all the ultra-cool dwarfs and at about the same critical temperature. Ackerman \& Marley (2001) treat the upward convective mixing of a gas, its condensation and the sedimentation of the condensate through the atmosphere of the object while Woitke \& Helling (2004) consider an ensamble of dust grains falling downwards from the top of the atmosphere. A detailed comparison of different atmospheric models of L dwarfs is presented in Helling et al. (2008).

The oblateness of a rotating object has been discussed by Chandrasekhar (2003) in the context of polytropic gas configuration under hydrostatic equilibrium. For a slow rotator, the relationship for the oblateness $f$ of a stable polytropic gas configuration under hydrostatic equilibrium is given by

$f=\frac{2}{3} C \frac{\Omega^{2} R_{\mathrm{e}}^{3}}{G M}$

where $M$ is the total mass, $R_{\mathrm{e}}$ is the equatorial radius, and $\Omega$ is the angular velocity of the object which is related to the linear velocity $V=\Omega \times R_{\mathrm{e}}$. $C$ is a constant whose value depends on the polytropic index. For a polytropic index of $n=1.0$, $C=1.1399$, which is appropriate for Jupiter (Hubbard 1984). For non-relativistic completely degenerate gas, $n=1.5$ and $C=0.9669$.

The effective temperature of the L dwarfs of different spectral type is determined by adopting a sixth order polynomial fit given by Golimowski et al. (2004) which is based on bolometric luminosities. The $T_{\text {eff }}$ calibration of Golimowski et al. (2004) agrees well in the interval L3-L8, but there are significant differences in earlier types. In our calculations for the degree of polarization, the effective temperature $T_{\text {eff }}$ is used and hence the degree of polarization should be considered strictly as a function of $T_{\text {eff }}$ rather than of spectral type. The mass and radius of the $\mathrm{L}$ dwarfs of different spectral types are estimated by adopting the empirical relationship given by Marley et al. (1996).

\section{Results}

We find a trend (more data are required to confirm our theory) of higher polarization in the $R$ band when compared to the $I$ band. 
Table 2. Polarization measurements.

\begin{tabular}{llllll}
\hline \hline Object & SpT & Filter & $\begin{array}{l}\text { Exposure time } \\
(\mathrm{s})\end{array}$ & $\begin{array}{l}\mathrm{D}(\mathrm{p}) \\
(\%)\end{array}$ & $\begin{array}{l}\theta \\
\text { (degrees) }\end{array}$ \\
\hline 2MASSW J1438082+640836 & M9.5 & $I$ & 90 & $0.482 \pm 0.071$ & 66.24 \\
& & $R$ & 300 & $0.54 \pm 0.097$ & 115 \\
2MASSW J1507476-162738 & L5 & $I$ & 150 & $0.0 \pm 0.036$ & $\ldots$ \\
& & $R$ & 300 & $0.216 \pm 0.022$ & 100.26 \\
2MASS J17312974+2721233 & L0 & $I$ & 60 & $5.195 \pm 0.854$ & 52.57 \\
& & $R$ & 240 & $0.666 \pm 0.169$ & 111.08 \\
2MASSI J1807159+501531 & L1.5 & $I$ & 60 & $0.711 \pm 0.142$ & 46.67 \\
& & $R$ & 120 & $1.669 \pm 0.605$ & 101.2 \\
\hline
\end{tabular}

Note: the uncertainty in $\theta$ due to calibration errors is about 1.2 degrees.

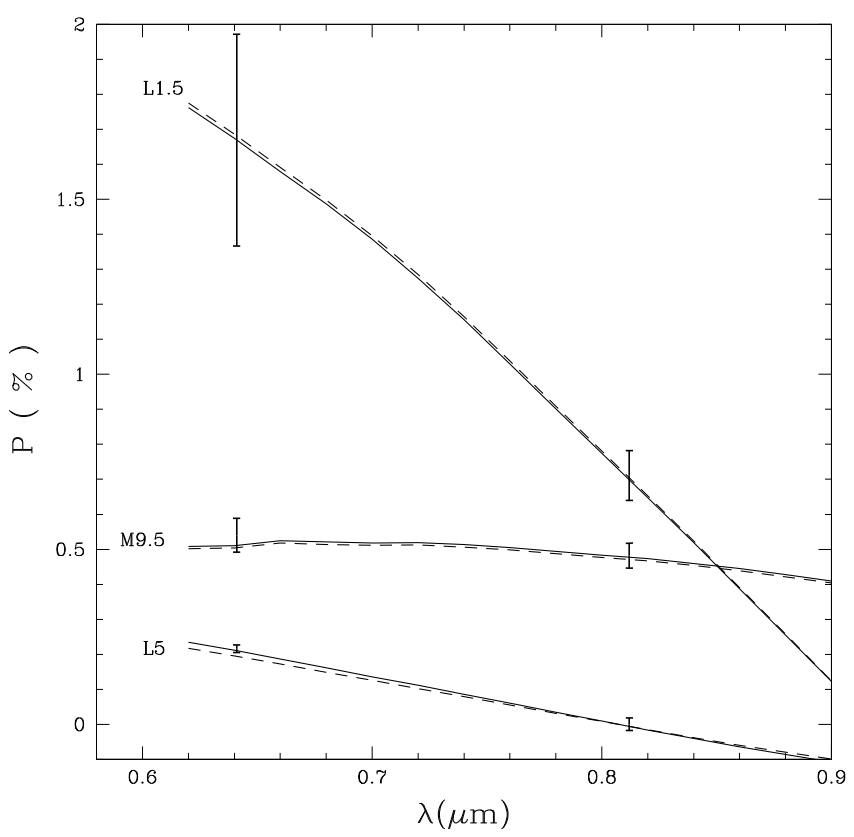

Fig. 1. Best model fit of the observed data. The solid lines represent the model with the polytropic index $n=1.0$ and dashed lines represent that with $n=1.5$. For other parameters see Table 3 .

This wavelength dependency strongly supports the argument by Sengupta \& Kwok (2005) that the polarization arises due to scattering and not because of magnetic field. In dust scattering as described by Mie theory, the amount of polarization depends on the ratio of the grain radius to the wavelength. For the same kind of dust species, the polarization usually peaks when the ratio is one. As a consequence, the increase in polarization with the decrease in wavelength implies the presence of sub-micron size grains in the photosphere of the L dwarfs. We present our measurements in Table 2, and our model fit in Fig. 1. One of the L dwarfs from Zapatero Osorio et al. (2005) (2MASSW J1507476-162738) shows a null polarization in our measurements in the $I$ band, whereas Zapatero Osorio et al. (2005) present a higher polarization $(1.36 \% \pm 0.30)$ for the same object. This suggests variability in linear polarization which in turn suggests atmospheric activities like dynamical variations of the cloud cover.

We also find relatively high polarization in the L0 dwarf. Additionally, from Schmidt et al. (2007), the $\mathrm{H} \alpha$ equivalent width is the highest for the L0 dwarf among the objects from our sample. This could be an indirect evidence of a disk around this ultra cool dwarf. We therefore searched the Spitzer public archive for mid-IR data. 2MASS J17312974+2721233 has been observed with IRAC and IRS in the course of program 3136

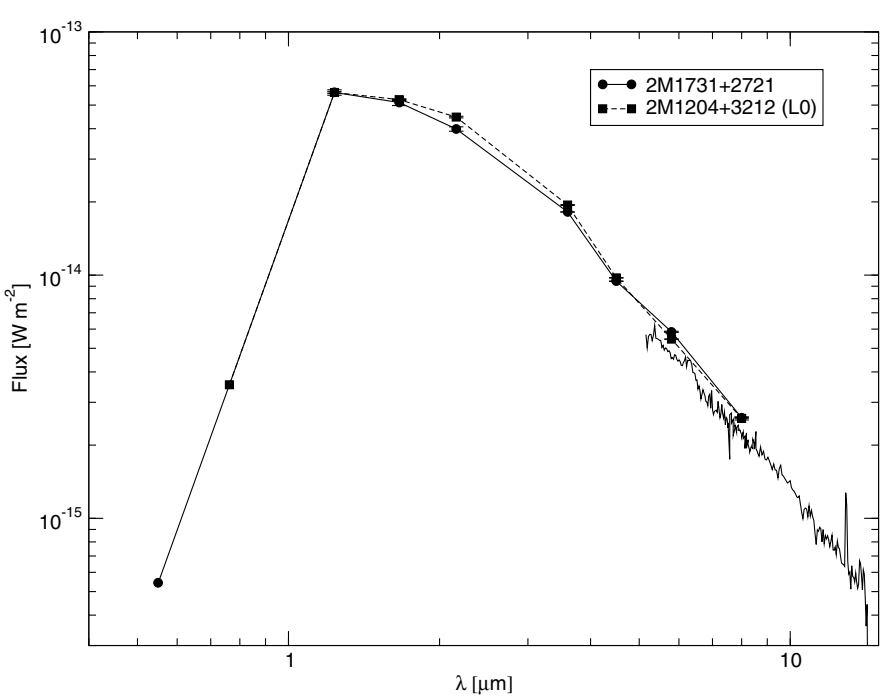

Fig. 2. Spectral energy distribution of 2MASS J17312974+2721233 (dots). $V$-band photometry from the LSPM-North proper-motion catalog of nearby stars (Lépine et al. 2005). $J, H$ and $K$ s photometry from 2MASS. IRAC and IRS photometry from this work. A comparison of L0 dwarf (2MASS J1204+3212, Patten et al. 2006) scaled to the same $J$-band flux is overplotted (squares). The target does not show any significant excess compared to the field L0 dwarf.

(P.I. Cruz), and we retrieved the pipeline processed data. We extracted the IRAC photometry using standard PSF photometry procedures within the Interactive Data Language. Uncertainties were estimated from the Poisson noise weighted by the coverage maps of the mosaics. Table 4 gives a summary of the photometry. Figure 2 shows the spectral energy distribution (SED) of the source and a L0 comparison object from the literature (2MASS J1204+3212, Patten et al. 2006). 2MASS J17312974+2721233 does not show any significant mid-IR excess up to $15 \mu \mathrm{m}$. The presence of a young circumstellar disc can therefore be ruled out at a high level of confidence. In the current state of the data, we cannot rule out the presence of a cold debris disc, as it would produce an excess at longer wavelengths.

The surface gravity of $\mathrm{L}$ dwarfs older than approximately a few hundred million years varies from $g=10^{5}$ to $3 \times 10^{5} \mathrm{~cm} \mathrm{~s}^{-2}$. Evolutionary models by Chabrier et al. (2000) show younger L dwarfs to have a surface gravity smaller than $10^{5} \mathrm{~cm} \mathrm{~s}^{-2}$. Our best fit model parameters are presented in Table 3 (best fit obtained by visual inspection). For the L dwarfs 2MASSW $\mathrm{J} 1438082+640836$, we find the best fit with $\log (g)=5.0$, and rotational velocity $V=18.7 \mathrm{~km} \mathrm{~s}^{-1}$ when $n=1.0$, and $V=20.5 \mathrm{~km} \mathrm{~s}^{-1}$ when $n=1.5$. For a fixed rotational velocity, the oblateness is lower when the polytropic index $n$ is 
Table 3. Model fit.

\begin{tabular}{llllll}
\hline \hline Object & SpT & $n$ & $\begin{array}{l}d_{0} \\
\text { (micron) }\end{array}$ & $\begin{array}{l}\log (g) \\
(\mathrm{cgs})\end{array}$ & $\begin{array}{l}V \\
\left(\mathrm{~km} \mathrm{~s}^{-1}\right)\end{array}$ \\
\hline 2MASSW J1438082+640836 & M9.5 & 1.0 & 0.7 & 5.0 & 18.7 \\
& & 1.5 & 0.7 & 5.0 & 20.5 \\
2MASSW J1507476-162738 & L5 & 1.0 & 0.44 & 5.255 & 27.2 \\
& & 1.5 & 0.44 & 5.230 & 27.2 \\
2MASSI J1807159+501531 & \multirow{2}{*}{ L1.5 } & 1.0 & 0.5 & 5.41 & 76.0 \\
& & 1.5 & 0.44 & 5.37 & 76.0 \\
\hline
\end{tabular}

Note: Bailer-Jones (2004) has measured the projected rotational velocity for 2MASSW J1507476-162738 as $27.2 \mathrm{~km} \mathrm{~s}^{-1}$, while Reiner \& Basri (2008) have reported the projected velocity for 2MASSW J1807159+501531 to be $76 \mathrm{~km} \mathrm{~s}^{-1}$.

Table 4. IRAC photometry of 2MASS J17312974+2721233.

\begin{tabular}{lc}
\hline \hline $\begin{array}{l}\text { Wavelength } \\
\text { (micron) }\end{array}$ & $\begin{array}{c}\text { Flux } \\
(\mathrm{mJy})\end{array}$ \\
\hline 3.6 & $21.82 \pm 0.02$ \\
4.5 & $14.16 \pm 0.02$ \\
5.8 & $11.30 \pm 0.06$ \\
8.0 & $6.91 \pm 0.03$ \\
\hline
\end{tabular}

higher. As a result, higher rotational velocity is needed to fit the observed data if $n=1.5$. The projected rotational velocity of the L dwarf 2MASSW J1507476-162738 is measured by Bailer-Jones (2004) while the projected rotational velocity of 2MASSW J1807159+501531 is estimated by Reiners \& Basri (2008). In the absence of any knowledge on the projection angle, we consider the minimum rotational velocity of these objects as $V=27.2 \mathrm{~km} \mathrm{~s}^{-1}$ and $V=76 \mathrm{~km} \mathrm{~s}^{-1}$. We find the best fit for the observed polarization of 2MASSWW J1507476162738 with $\log (g)=5.255$ for $n=1.0$ and $\log (g)=5.230$ for $n=1.5$ For 2MASSW J1807159+501531 the best fit is obtained with $\log (g)=5.41$, when $n=1.0$, and $\log (g)=5.37$ when $n=1.5$. Note that the oblateness decreases with the increase in surface gravity. For all the cases, the observed polarization profiles can be fitted with sub-micron size grains, and the mean size of grains that are required to fit the observation is consistent with the recent theoretical calculations of dust properties (Woitke \& Helling 2004; Woitke \& Helling 2003). Polarization measurements for one of the above three objects (2MASSW J1507476162738) were also recently published Goldman et al. (2009). Our results are consistent with the Goldman et al. (2009) measurements within $1 \sigma$ error bars for this object. This work differs from Goldman et al. (2009) in the aspect that our sample could reproduce the predictions of Sengupta \& Kwok (2005) whereas the Goldman et al. (2009) sample does not. Both the studies (this paper and Goldman et al. 2009) have sparse statistics, which discourages us to make any speculations on this apparent discrepancy.

\section{Conclusions}

1. We report linear polarizaion measurements of 4 very nearby ultra cool dwarfs in the $R$ and $I$ bands.

2. We find that there is a trend (3 out of 4 ) of a higher degree of polarization at shorter wavelengths ( $R$ band) when compared to the $I$ band as predicted by the theoretical models of Sengupta \& Kwok (2005).

3. The L0 dwarf 2MASS J17312974+2721233 is interesting because of its relatively high polarization and requires follow-up studies.
4. We also fit theoretical models to predict the dust grain size and rotational velocities of three of the ultra cool dwarfs.

5. We find evidence for variability in the linear polarization for (2MASSW J1507476-162738). This suggests atmospheric activities like dynamical variations of the cloud cover in this object.

Acknowledgements. This work was partially funded by the Spanish MICINN under the Consolider-Ingenio 2010 Program grant CSD2006-00070: First Science with the GTC (http://www.iac.es/consolider-ingenio-gtc)

\section{References}

Ackerman, A. S., \& Marley, M. S. 2001, ApJ, 556, 872

Allard, F., Hauschildt, P. H., Alexander, D. R., Tamanai, A., \& Schweitzer, A. 2001, ApJ, 556, 357

Bailer-Jones, C. A. L. 2004, A\&A, 419, 703

Basri, G., Mohanty, S., Allard, F., et al. 2000, ApJ, 538, 363

Berger, E., Rutledge, R. E., Reid, I. N., et al. 2005, ApJ, 627, 960

Burrows, A., \& Sharp, C. M. 1999, ApJ, 512, 843

Burrows, A., Hubbard, W. B., Lunine, J. I., \& Liebert, J. 2001, RvMP, 73, 719

Burrows, A., Sudarsky, D., \& Hubeny, I. 2006, ApJ, 640, 1063

Chabrier, G., Baraffe, I., Allard, F., \& Hauschildt, P. 2000, ApJ, 542, 464

Chandrasekhar, S. 1933, MNRAS, 93, 539

Chiu, K., Fan, X., Leggett, S. K., et al. 2006, AJ, 131, 2722

Cooper, C. S., Sudarsky, D., Milsom, J. A., Lunine, J. I., \& Burrows, A. 2003, ApJ, 586, 1320

Cruz, K. L., Reid, I. N., Liebert, J., Kirkpatrick, J. D., \& Lowrance, P. J. 2003, AJ, 126, 2421

Goldman, B., Pitann, J., Zapatero Osorio, M. R., et al. 2009, A\&A, 502, 929 Golimowski, D. A., Leggett, S. K., Marley, M. S., et al. 2004, AJ, 127, 3516 Helling, Ch. 2003, Rev. Mod. Astron. 16, 15

Helling, Ch., Ackerman, A., Allard, F., et al. 2008, MNRAS, 391, 1854

Hubbard, W. B. 1984, Planetary Interiors (New York: Van Nostrand Reinhold)

Jameson, et al. 2007, AJ, 119, 339

Kirkpatrick, J. D., Allard, F., Bida, T., et al. 1999, ApJ, 519, 834

Knapp, G. R., Leggett, S. K., Fan, X., et al. 2004, AJ, 127, 3553

Marley, M. S., Saumon, D., Guillot, T., et al. 1996, Science, 272, 1919

Ménard, F., Delfosse, X., Monin, J.-L., et al. 2002, A\&A, 396, L35

Patten, B. M., Stauffer, J. R., Burrows, A., et al. 2006, ApJ, 651, 502

Reid, I. N., Kirkpatrick, J. D., Gizis, J. E. et al. 2000, AJ, 119, 369

Reiners, A., \& Basri, G. 2008, ApJ, 684, 1390

Schmidt, S. J., Cruz, K. L., Bongiorno, B. J., Liebert, J., \& Reid, I. N. 2007, AJ, 133,2258

Sengupta, S., \& Krishan, V. 2001, ApJ, 561, L123

Sengupta, S., \& Krishan, V. 2003, ApJ, 585, L155

Sengupta, S., \& Kwok, S. 2005, ApJ, 625, 996

Tsuji, T., \& Nakajima, T. 2003, ApJ, 585, L151

Tsuji, T., Ohnaka, K., Aoki, W., \& Nakajima, T. 1996, A\&A, 308, L29

Tsuji, T., Nakajima, T., \& Yanagisawa, K. 2004, ApJ, 607, 511

van de Hulst, H. C. 1957, Light Scattering by Small Particles (New York: Willey)

Woitke, P., \& Helling, Ch. 2003, A\&A, 399, 297

Woitke, P., \& Helling, Ch. 2004, A\&A, 414, 335

Zapatero Osorio, M. R., Caballero, J. A., \& Béjar, V. J. S. 2005, ApJ, 621, 445 